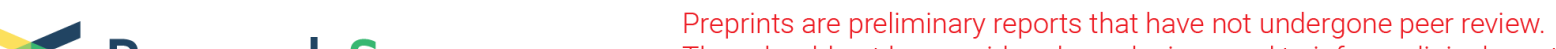 Research Square
or referenced by the media as validated information.
}

\section{Record performance of ultralow-temperature-driven water-based sorption refrigeration enabled by low- cost zeolite-like porous aluminophosphate}

\section{Zhang Li liu}

Institute of Engineering Thermophysics, Chinese Academy of Sciences

Jiaxing Xu

Shanghai Jiao Tong University

Xu Min ( $\nabla$ xumin@iet.cn )

Institute of Engineering Thermophysics, Chinese Academy of Sciences https://orcid.org/0000-0003-

4033-9204

\section{Caifeng Huang}

Institute of Engineering Thermophysics, Chinese Academy of Sciences

\section{Ruzhu Wang}

Shanghai Jiao Tong University https://orcid.org/0000-0003-3586-5728

\section{Tingxian Li}

Shanghai Jiao Tong University https://orcid.org/0000-0003-4618-8144

\section{Xiulan Huai}

Institute of Engineering Thermophysics, Chinese Academy of Sciences

\section{Article}

Keywords: refrigeration, sorption, chlorofluorocarbon refrigerant

Posted Date: July 26th, 2021

DOI: https://doi.org/10.21203/rs.3.rs-688181/v1

License: (c) (1) This work is licensed under a Creative Commons Attribution 4.0 International License.

Read Full License

Version of Record: A version of this preprint was published at Nature Communications on January 11th, 2022. See the published version at https://doi.org/10.1038/s41467-021-27883-4. 


\section{Abstract}

Thermally driven water-based sorption refrigeration is considered a promising strategy to realize nearzero-carbon cooling applications by addressing the urgent global climate challenge caused by conventional chlorofluorocarbon (CFC) refrigerants. However, developing cost-effective and highperformance water-sorption porous materials driven by low-temperature thermal energy is still a significant challenge. Here, we propose a low-cost zeolite-like aluminophosphate with SFO topology (EMM-8) for water-sorption-driven refrigeration. The EMM-8 is characterized by 12-membered ring channels with large accessible pore volume and exhibits high water uptake of $0.36 \mathrm{~g} \cdot \mathrm{g}^{-1}$, low-temperature regeneration of $65^{\circ} \mathrm{C}$, fast adsorption kinetics, remarkable hydrothermal stability, and scalable fabrication. Importantly, the water-sorption-based chiller with EMM-8 shows the potential of achieving a record coefficient of performance (COP) of 0.85 at an ultralow-driven temperature of $63^{\circ} \mathrm{C}$. The extraordinary working performance makes EMM-8 a practical alternative to realize high-efficient ultra-lowtemperature-driven refrigeration.

\section{Introduction}

Energy demands for heating and cooling account for more than $50 \%$ of global final energy consumption and are expected to maintain a rapidly increasing rate in the future decades, especially for cooling purposes ${ }^{1-4}$. Accordingly, carbon dioxide $\left(\mathrm{CO}_{2}\right)$ emissions related to heating and cooling have grown significantly since most of the heat and cold is produced by fossil fuels at present ${ }^{1,5,6}$. Therefore, it is urgent to shift heating and cooling to be a more low-carbon process. Water-based adsorption heat pumps (AHPs) and adsorption chillers (ADCs) (see the working principle in Supplementary Fig. 1 and Note 1), driven by sustainable low-grade thermal energy, are considered as potential alternatives to mitigate climate change and fuels shortage $\mathrm{e}^{7-10}$. Moreover, the utilization of natural refrigerants of water in adsorption systems is more environmental-friendly than fluorocarbon type refrigerants in vapor compression systems. Thus, near-zero-carbon-emission adsorption heating and cooling have triggered a new round of worldwide research to meet the fast-growing energy demands and face the vast challenges of global warming and environmental pollution in recent decades.

For water-sorption-driven heat pumps and chillers, adsorbents are the critical factor in determining the performance of systems ${ }^{11}$. The perfect water adsorbents should exhibit the following characters: i) Sshaped water adsorption isotherms and superior water uptakes; ii) low regeneration temperatures; iii) excellent hydrothermal stabilities; iv) fast water adsorption and desorption kinetics; v) facile and low-cost synthesis. Traditional water adsorbents, such as silica gels ${ }^{12,13}$ and zeolites ${ }^{14,15}$, have been extensively investigated for adsorption heating and cooling systems. Despite they show advantages of acceptable price and desirable stabilities, obvious drawbacks are identified, including high regeneration temperatures ( $>150{ }^{\circ} \mathrm{C}$ for zeolites) and low working capacities, resulting in poor energy efficiency and low power density for adsorption systems. Alternatively, metal-organic frameworks (MOFs) with highly tunable pore structures and hydrophilic/hydrophobic functional groups, are considered as new effective water 
adsorbents $^{16-18}$. During the last decades, lots of new MOFs with high water adsorption capacities have been reported, wherein Zr- and Al-based MOFs, such as MOF-80 ${ }^{19}{ }^{19}$ MOF-303 ${ }^{20}$, MIP-200 21 , MIL-160 22 , CAU-23 ${ }^{23}, \mathrm{Zr}$-furm ${ }^{24}$, and KMF-1 ${ }^{25}$, gain much attention due to their notably hydrothermal stability. Among them, KMF-1 and MIP-200 show high energy efficiency for both heating and cooling applications. The lab-scale ADC prototypes using MOFs have also been recently demonstrated ${ }^{26,27}$. However, although many attempts to realize commercial-scale production are reported ${ }^{28,29}$, challenges from expensive raw materials and low-scalability in synthesis make MOFs unaffordable in this stage ${ }^{7}$. Consequently, it is exceedingly essential to exploit novel water adsorbents to achieve a breakthrough in both low-cost and high-performance.

Aluminophosphate molecular sieves (AIPOs), a family of zeolite-derivatives-based porous materials, show comparable low cost in synthesis and reasonable market availability for AHP and ADC applications. For example, SAPO-34, usually be used as a catalyst for menthol to olefin (MTO) reaction, has been investigated for more than two decades as a promising water adsorbent ${ }^{30,31}$. Several years ago, the company of Mitsubishi Plastics from Japan developed two types of aluminophosphates (namely AQSOA-Z01 and AQSOA-Z02), representing a considerable advancement for ADC applications owing to their outstanding performances of high water uptake and low regeneration temperature $\left(<90^{\circ} \mathrm{C}\right)^{32-34}$. The attractive performances of lab-scale and commercial-scale ADCs using AQSOA-ZO2 have been demonstrated ${ }^{35,36}$. Recently, a small-pore aluminophosphate AIPO-LTA was reported as a promising candidate for water-sorption-based heat pump and thermal energy storage ${ }^{37}$. Moreover, aluminophosphates deliver another advantage of considerable industrial-production capacity. For instance, a SAPO-34 manufacturing plant with the production capacity of 5000 tons per year in Dalian was built in $2018^{38}$. Unfortunately, the AIPOs reported so far cannot hold a candle to the best current MOFs, especially in terms of the performance of ultra-low-temperature-driven chillers.

In this work, we search out an SFO-type aluminophosphate with superior performance, namely EMM-8, mainly built of 12-membered ring channels. The accessible pore volume of EMM-8 (19.57\%) is larger than that of usually-used SAPO-34 (17.27\%) and AIPO-5 (14.07\%) according to the database of zeolite structures, suggesting potential better water adsorption capacity. Also, the diameter of a 12-ring window for EMM-8 is $\sim 0.7 \mathrm{~nm}$, larger than that of 8-ring openings for SAPO-34 and AIPO-LTA $(\sim 0.4 \mathrm{~nm})$, indicating weaker hydrophilicity and easy desorption of water molecules at a lower driven temperature. Remarkably, this EMM-8 can be synthesized based on very cheap and common raw materials, pseudo-boehmite and phosphoric acid. A wildly-used and scalable compound, 4dimethylaminopyridine (4-DMAPy), is exploited as a structure-directing agent (SDA), making the synthesis of EMM-8 further low-cost. Although the structure of EMM-8 was reported in $2007^{39}$, its potential in waterbased applications has not been exploited to date as a result of insufficient investigations on materials synthesis, characterizations, and rare evaluations on gas sorption performance.

Herein, we report the first-ever use of EMM-8 as an efficient water adsorbent for ultra-low-temperaturedriven heating and cooling. We thoroughly characterize it towards water adsorption application and 
achieve a $100 \mathrm{~g}$-scale fabrication. By comprehensive water sorption evaluation, we find the EMM-8 represents step-wise water adsorption at $P / P_{0}=\sim 0.15$, facile desorption at low temperature $\left(\sim 65^{\circ} \mathrm{C}\right)$, and desirable hydrothermal stability. More importantly, EMM-8 shows a notably high COP of 0.85 (refrigeration) and 1.75 (heat pump) at lower driven temperatures than those of the best current state-ofthe-art materials by $5-15^{\circ} \mathrm{C}$, indicating a promising application potential for the exploitation of yet mostly unused low-grade heat. To elaborate the mechanism of high efficiencies at ultralow temperatures, we carried out the theoretical simulation together with experimental measurements, uncovering the high COP originates from a considerably low isosteric heat of adsorption contributed by the hydrogen-bonded water clusters within the pores of EMM-8. This work provides a more effective water adsorbent for realizing affordable, scalable, and high-performance adsorption heating and cooling.

\section{Results And Discussion}

\section{Characterization and scalable synthesis}

We prepared EMM-8 with hydrothermal synthesis according to the method reported by Afeworki et al. ${ }^{40} \mathrm{X}$ ray diffraction (XRD) measurements (Fig. 1a) confirms that the prepared aluminophosphate possesses the SFO topological structure. As shown in Figure $1 \mathrm{~b}$ and Supplementary Fig. 2, the basic building units of EMM-8 are double four-rings (D4Rs), which form a two-dimensional framework with 12-ring and 8-ring channels. The ${ }^{27} \mathrm{Al}$ and ${ }^{31} \mathrm{P}$ magic-angle spinning (MAS) NMR spectrums (Fig. 1c) and X-ray photoelectron spectroscopy (XPS) measurements (Supplementary Fig. 3) of the calcined EMM-8 also confirm the tetrahedrally coordinated framework with $\mathrm{Al}$ and $\mathrm{P}$ atoms. Nitrogen adsorption results also show the EMM-8 has a high BET surface area of $879.63 \mathrm{~m}^{2} \cdot \mathrm{g}^{-1}$ and a large pore volume of $0.59 \mathrm{~cm}^{3} \cdot \mathrm{g}^{-1}$ (Fig. 1d and Supplementary Table 1). Moreover, we find a concentrated pore diameter distribution with an average micropore size of $0.7 \mathrm{~nm}$, corresponding to the size of 12-ring windows. The nitrogen adsorption isotherm also confirms the presence of the mesopores, whose volume is as high as $0.288 \mathrm{~cm}^{3} \cdot \mathrm{g}^{-1}$ that is close to that of micropores.

SEM and TEM images show two-edged sword-shaped and highly faceted EMM-8 particles (Fig. 2a-c), indicating the good crystallinity and high purity of synthesized samples. High-resolution TEM image (Fig. $2 \mathrm{~d}$ ) of the material shows clear lattice fringes, and selective area electron diffraction (SAED) pattern (Fig. $2 e)$ clearly suggests its single crystal characteristics. Moreover, we find the zeolite-like crystals have uniform shapes in length $(1-3 \mu \mathrm{m})$ and width (200-300 nm). SEM and AFM observations (Fig. $2 \mathrm{~b}$ and fh) reveal that the thicknesses of the EMM- 8 nanoplates are distributed between $20 \mathrm{~nm}$ and $120 \mathrm{~nm}$. The gap between the adjacent nanoplates contributes to the presence of mesopores. It is expected that the nano-sized plate-like structures with abundant intervals offer a high potential to achieve a faster transfer of water molecules within interparticle spaces and micropores.

For the water-sorption-based heating and cooling applications, it is crucial that the adsorbent has hydrothermally stable pores. Temperature-dependent powder XRD (Fig. 1a) and thermogravimetric analysis (TGA) (Supplementary Fig. 4) reveal the total removal of the SDA at $\sim 400{ }^{\circ} \mathrm{C}$ and demonstrate 
the desirable thermal stability of the synthesized EMM-8 up to $700{ }^{\circ} \mathrm{C}$, which is much better than that of MOFs. Also, we performed the hydrothermal stability tests by soaking the calcined EMM-8 in boiling water for $24 \mathrm{~h}$, and the results point out that the obtained sorbent can maintain its crystallinity, framework structure, and microporosity, suggesting its stability under harsh working conditions. This conclusion was further evidenced by the NMR spectrum (Fig. 1c), XRD patterns, and $\mathrm{N}_{2}$ adsorption isotherms (Supplementary Fig. 5).

To build a real-life full-scale ADC device, it is imperative that the sample synthesis process of water adsorbents should be easily scaled up. Thus, the hundred-gram-scale production of this material is performed by a scaled-up reactor in this work. As shown in Supplementary Fig. 6a, we use a $2 \mathrm{~L}$ Teflon vessel to replace the $50 \mathrm{~mL}$ one in synthesis and introduce 40 times the number of raw materials in it. As a result, about $100 \mathrm{~g}$ EMM-8 with good crystallinity is obtained, suggesting the robust fabrication of this aluminophosphate (Supplementary Fig. 6b). Importantly, it is found that there is no significant difference between crystals of EMM-8 synthesized by $50-\mathrm{mL}$ and 2-L Teflon vessels, evidenced by SEM images, XRD patterns, and $\mathrm{N}_{2}$ adsorption isotherms (Supplementary Fig. 7a-c). Moreover, it is worth mentioning that EMM-8 is easily shaped as pellets by pressing without any binders (Supplementary Fig. 7d).

\section{Water adsorption evaluation}

The water adsorption isotherms of EMM-8 were measured by a gravimetric adsorption analyzer at three different temperatures $\left(25,40\right.$, and $50^{\circ} \mathrm{C}$ ) (Fig. 3a). The results represent a perfect S-shaped water adsorption isotherm with a step-wise water uptake in the extremely narrow relative pressure range of $P / P_{0}=0.15-0.17$, indicative of the presence of uniform micropores. EMM-8 exhibits a relatively high water adsorption capacity of $0.283 \mathrm{~g}_{\mathrm{H} 20^{\circ}} \mathrm{g}_{\text {sorbent }}{ }^{-1}$ at $30{ }^{\circ} \mathrm{C}$ and $P / P_{0}=0.3$, comparable to that of the selected state-of-the-art water adsorbents (Supplementary Fig. 8), including SAPO-34, MOF-801, CAU-10, COF-TpPa ${ }^{41}$, and Co-CUK-1 ${ }^{42}$.

A calculated average isosteric enthalpy of water adsorption was determined to be $46.76 \mathrm{~kJ} \cdot \mathrm{mol}^{-1}$ (Fig. $3 \mathrm{~b}$ ) by using the Clausius-Clapeyron equation based on the data of water adsorption isotherms at three different temperatures. We found this value of EMM-8 is lower than that of many other benchmark sorbents (Fig. 3b and Supplementary Table 3) and merely higher than the evaporation enthalpy of water $\left(44 \mathrm{~kJ} \cdot \mathrm{mol}^{-1}\right)$, indicating a relatively lower energy consumption for water desorption and thus higher COP for sorption-based heating or cooling system. Unlike the strong electrostatic interaction between the framework and water molecules for conventional zeolite, the zeotypic EMM-8 with electrical neutrality endows a weak hydrogen-bonded network of water molecules within micropores, leading to lower adsorption enthalpy, which will be detailed in the following section. The characteristic curves defining the relations of adsorption potential and water uptake ${ }^{16}$ are determined using adsorption isotherms of EMM8 and other reference materials, as shown in Fig. 3c. In the context of meeting the typical operating conditions for both refrigeration and heat pump applications, EMM-8 exhibits a significantly lower 
adsorption potential than conventionally-used zeolite, SAPO-34, and Co-CUK-1, indicating the lower required driving temperature.

In order to further confirm the suitability of the material for applications on ADC and AHP, cycling stability tests were carried out under the condition of regeneration temperature of $110^{\circ} \mathrm{C}$ and adsorption temperature of $30^{\circ} \mathrm{C}$. The results reveal no significant loss of the water uptake for EMM-8 over 30 adsorption/desorption cycles (Fig. 3d).

To further explore the adsorption mechanism at the microscopic level, the water adsorption isotherm of EMM-8 was calculated by using Grand Canonical Monte Carlo (GCMC) simulations at $30^{\circ} \mathrm{C}$. The obtained result matches the experimental adsorption isotherm well, as shown in Supplementary Fig. 9. At a low relative pressure of $P / P_{0}=0.01$, only a small amount of water molecules enter into the 12-ring channels, associated with a high adsorption enthalpy $\left(71 \mathrm{~kJ} \cdot \mathrm{mol}^{-1}\right.$, Supplementary Fig. 10). The water molecules are preferentially coordinated to aluminum atoms on the pore wall (Fig. 4a), resulting in the change of the aluminum atom from tetrahedral coordination to octahedral coordination. This phenomenon is very common for water adsorption on AlPOs ${ }^{39,43-45}$ and consistent with the ${ }^{27} \mathrm{Al} \mathrm{NMR}$ experimental findings (Supplementary Fig. 11a). When the relative water vapor pressure is above 0.25 , there is a sudden increase in the water adsorption capacity and the water molecules tend to fill the entire 12-ring 1D pore openings of EMM-8, as shown in Fig. 4b and c. It also can be seen that a hydrogen bond network of water molecules is formed with a total number of hydrogen bonds per water molecule of 2.9 (Supplementary Fig. 12), which is close to that of bulk water. This observation is supported by the ${ }^{1} \mathrm{H}$ NMR spectra (Supplementary Fig. 11b), which shows the low-field shift of the water signal, indicating the presence of the hydrogen-bonded water molecules. This low-energy water-water molecular interaction suggests a low enthalpy of adsorption $\left(40-50 \mathrm{~kJ} \cdot \mathrm{mol}^{-1}\right.$, Supplementary Fig. 10) and thus results in the facile regeneration of EMM-8.

\section{ADC performance evaluation}

Materials-based COPs for cooling, which are usually used to assess the energy efficiency of the ADC devices, are evaluated according to the methodology by de Lange et al. ${ }^{18}$ (Supplementary Note 2 ) at different boundary temperatures for evaporation $\left(T_{\mathrm{ev}}\right)$, condensation $\left(T_{\text {con }}\right)$, and regeneration $\left(T_{\text {des }}\right)$. Figure 5a shows the calculated maximum $\mathrm{COP}_{\mathrm{C}}$ for $\mathrm{EMM}-8$ and its corresponding driving temperature for a specific refrigeration condition, i.e., $T_{\mathrm{ev}}=5^{\circ} \mathrm{C}$ and $T_{\text {con }}=30^{\circ} \mathrm{C}$. The results indicate that EMM- 8 exhibits an exceptionally high $\mathrm{COP}_{\mathrm{C}}$ of 0.85 at the ultralow driving temperature of $63^{\circ} \mathrm{C}$, remarkably exceeding the existing state-of-the-art materials, including the recently reported best-in-class adsorbents, such as AIPOLTA (0.75), MIP-200 (0.78), KFM-1 (0.75), and Co-CUK-1 (0.83). Additionally, the very high thermal efficiencies and working capacities on cooling retain for a wide range of evaporation temperatures, as illustrated in Supplementary Fig. 13a and 14. More importantly, the required driving temperatures, achieving the highest COP and working capacity of EMM-8, are lower than those of reference materials by $5-15^{\circ} \mathrm{C}$, as shown in Fig. $5 a$ and b, which is significantly meaningful for the efficient exploitation of ultra- 
low-grade thermal energy. The complementary metric, specific energy capacity, i.e., the provided cooling capacity from the evaporator in one cooling cycle $\left(Q_{\mathrm{ev}}\right)$, is evaluated and compared with that of other benchmark materials (Supplementary Fig. 15). The excellent volumetric specific energy capacity for EMM-8 is also found even at a low regeneration temperature of $65^{\circ} \mathrm{C}$ (Fig.5c and Supplementary Table 3 ), which is another indication of the extraordinary cooling performance of EMM-8 at ultralow driving temperatures.

Moreover, under a standard heat pump condition, i.e., $T_{\mathrm{ev}}=15^{\circ} \mathrm{C}$ and $T_{\text {con }}=45^{\circ} \mathrm{C}$, EMM-8 also gives a very high $\mathrm{COP}_{\mathrm{H}}$ of 1.75 at a driven temperature of $82^{\circ} \mathrm{C}$ (Fig. $5 \mathrm{~d}$ and Supplementary Fig. 13b). The comparison of this value between EMM-8 and other excellent materials confirms that EMM-8 outperforms both other AIPOs and most MOFs for heat pump applications. This value is also as high as that of the best water adsorbents reported so far, i.e., Co-CUK-1 (1.77) and KMF-1 (1.74). However, in comparison with Co-CUK-1, EMM-8 shows higher volumetric working capacities at lower desorption temperatures (Fig. $5 e$ ). To the best of our knowledge, therefore, the obtained unprecedentedly high cooling and heating efficiencies suggest that EMM-8 is one of the best water adsorbents to date for realizing ultra-lowtemperature-driven ADC and AHP applications.

Alongside COP, the specific cooling power (SCP) of the water adsorbent is also a key performance indicator that dictates the power density of ADC devices. EMM-8 was shaped via pressing, crushing, sieving, and then deposited on a flat-plate adhesive plate (Supplementary Fig. 17b) to assess its dynamic sorption performances under varying ADC operating conditions (Supplementary Table 5). The water desorption profiles of EMM-8 show the fast desorption rates under the operating conditions of $T_{\text {con }}=30$ ${ }^{\circ} \mathrm{C}$, and $T_{\text {des }}=65^{\circ} \mathrm{C}$ (Fig. 5f), demonstrating its superiority in the deep utilization of ultra-low-temperature heat. According to the evaluations of SCPs based on kinetic measurements (Supplementary Note 3), EMM-8 has significantly high power density in ADCs operated at low evaporator temperature $\left(5-10{ }^{\circ} \mathrm{C}\right)$ and low driven temperatures $\left(65-80^{\circ} \mathrm{C}\right)$, as seen in Supplementary Table 6 and 7. Typically, even under the rather harsh working conditions $\left(T_{\mathrm{ev}}=5^{\circ} \mathrm{C}, T_{\text {con }}=30^{\circ} \mathrm{C}\right.$, and $\left.T_{\text {des }}=65^{\circ} \mathrm{C}\right)$, a superior high $\mathrm{SCP}_{\max }$ of $2.22 \mathrm{~kW} \cdot \mathrm{kg}_{\text {sorbents }}{ }^{-1}$ and $\mathrm{SCP}_{80 \%}$ of $1.1 \mathrm{~kW} \cdot \mathrm{kg}_{\text {sorbents }}{ }^{-1}$ for this material with the size of $0.45-0.6 \mathrm{~mm}$ is achieved. The $\mathrm{SCP}_{80 \%}$ value of EMM-8 surpasses those of both commercial water sorbents and promising MOFs (Supplementary Table 8), suggesting a unique opportunity to construct a compact and lightweight sorption system using this adsorbent.

The production cost of water sorbents is also a key concern for the application of the ADC systems. The cost of reagent-grade raw materials for EMM-8 is one percent to one third of the outstanding MOFs considered here (Supplementary Table 9), indicating its extremely attractive advantage in commercialization potentials. Accordingly, together with its outperforming energy efficiency, power density, and level of production maturity, the proposed EMM-8 clearly shows a successful combination of superior performance and economic potential.

\section{Conclusion}


This work demonstrates a zeotypic aluminophosphate EMM-8 as a high-performance water adsorbent that outperforms all the previously reported MOFs for ultra-low-temperature-driven refrigeration applications. EMM-8 exhibits excellent water adsorption performances of high water uptake at an extremely narrow relative pressure range, fast sorption kinetics, facile regeneration, and remarkable stability. The abundant micropores with a uniform pore size of $0.7 \mathrm{~nm}$ and low water adsorption enthalpy endow adsorption refrigeration systems with an unprecedentedly high COP of 0.85 at a low driving temperature of $63^{\circ} \mathrm{C}$. Moreover, the adsorption -based system using EMM-8 shows a potential of high $\mathrm{SCP}_{80 \%}$ of $1.1 \mathrm{~kW} \cdot \mathrm{kg}_{\text {sorbents }}{ }^{-1}$ under rigorous cooling conditions at the evaporation temperature as low as $5{ }^{\circ} \mathrm{C}$ and desorption temperature as low as $65^{\circ} \mathrm{C}$. The developed EMM- 8 is expected to be a promising candidate for adsorption-based refrigeration, featuring extraordinary working performance and desirable stability, combined with unique advantages of low-cost and scalable synthesis. This work paves a lowcarbon way of developing a new promising aluminophosphate adsorbent to realize energy-efficient ultralow-temperature-driven refrigeration.

\section{Methods}

\section{Typical synthesis of EMM-8}

The aluminum precursor, $0.662 \mathrm{~g}$ pseudo-boehmite, was slowly added to a mixture solution of $3.6 \mathrm{ml}$ deionized water and $0.682 \mathrm{ml} 85 \%$ phosphoric acid. The solution was followed by strong stirring at room temperature for $10 \mathrm{~min}$ to form a homogeneous suspension. Then, $0.112 \mathrm{ml}$ of mineralizer $40 \% \mathrm{HF}$ and $1.234 \mathrm{~g}$ 4-dimethylaminopyridine were added to the mixture. After another $10 \mathrm{~min}$ of stirring and $10 \mathrm{~min}$ of ultrasonication, the gel was transferred to a $50 \mathrm{ml}$ Teflon-lined stainless-steel autoclave. The reactor was heated and kept at $175^{\circ} \mathrm{C}$ for $72 \sim 84 \mathrm{~h}$ under static conditions. After cooling down to room temperature, the white powder of EMM-8 was collected by centrifuged, washed with deionized water, and dried in a vacuum oven. The as-synthesis samples were calcined directly at $600{ }^{\circ} \mathrm{C}$ for $6 \mathrm{~h}$ with heating rates of 5 ${ }^{\circ} \mathrm{C} \cdot \mathrm{min}^{-1}$ to remove the SDA.

\section{Characterization}

The powder X-ray diffraction (XRD) patterns were recorded at room temperature under ambient conditions with a BRUKER instrument (D8 Focus, Cu K $\mathrm{a}_{\mathrm{a}}$ with $\mathrm{k}=1.5418 \AA$ ). The morphologies of samples were characterized by scanning electron microscopy (SEM, Hitachi S4800) and higher resolution transmission electron microscopy (HRTEM, JEM-2100F) equipped with an EDX detector. The surface morphology and thickness of the sample were analyzed by atomic force microscope (AFM, Bruker Multimode 8) using dynamic mode scanning at scan area $2 \times 2 \mu \mathrm{m}^{2}$. Specific surface area and pore volume of samples were obtained by nitrogen gas adsorption at a low temperature of about $77 \mathrm{~K}$ using a gas adsorption analyzer (Quantachrome Quadrasorb SI-MP). Solid-state NMR spectra were recorded with an Advance III HD Bruker 500 NMR spectrometer $\left(B_{0}=11.7 \mathrm{~T}\right.$, which corresponds to Larmor frequencies of $500.1,130$, and $202 \mathrm{MHz}$ for ${ }^{1} \mathrm{H},{ }^{27} \mathrm{Al}$, and ${ }^{31} \mathrm{P}$, respectively). The samples were packed either in $4 \mathrm{~mm}$ (for ${ }^{27} \mathrm{Al}$ and ${ }^{31} \mathrm{P}$, MAS 10 and $13.3 \mathrm{kHz}$ ) and $2.5 \mathrm{~mm}$ (for ${ }^{1} \mathrm{H}$, MAS $30 \mathrm{kHz}$ ) outer diameter rotors. The surface 
composition was measured by X-ray photoelectron spectroscopy (XPS, ESCALAB 250Xi). Inductively coupled plasma optical emission spectroscopy (ICP-OES) analysis was carried out on a Thermo (iCAP Q) spectrometer. The structural stability of the samples was characterized by a thermogravimetric analyzer (TGA, TG-DTA6300).

\section{Water sorption measurement}

Water sorption isotherms were measured by a 3H-2000 PW intelligent gravimetric analyzer (IGA, Beishide Instrument Technology Co., Ltd.). The IGA was automatically operated to precisely control the water vapor pressure $(1 \sim 95 \% \mathrm{RH})$ and temperature $\left(20 \sim 60^{\circ} \mathrm{C}\right)$. Prior to adsorption experiments, samples were completely dehydrated at $150{ }^{\circ} \mathrm{C}$ under vacuum.

\section{Cyclic water adsorption/desorption tests}

Multiple cycles of water adsorption-desorption were also performed using a $3 \mathrm{H}-2000 \mathrm{PW}$ intelligent gravimetric analyzer. The weight-gain profiles were measured at $30{ }^{\circ} \mathrm{C}$ and $30 \% \mathrm{RH}$, while the weight-loss ones were collected at $110^{\circ} \mathrm{C}$ under vacuum.

\section{Kinetic measurements}

The water sorption kinetics of sorbents were measured by using a self-constructed testing system based on the method proposed by Aristov ${ }^{46,47}$ (Supplementary Fig. 16). The EMM-8 grains were obtained by powder pressing without binder, manual grinding, and sieving, successively. The shaped EMM-8 granules with different sizes of 0.3-0.45 mm, 0.45-0.6 mm, and 0.8-0.9 mm (Supplementary Fig. 17, 18 and Table 4) were used in this work. The characteristic sorption time ( $\tau$ ), as well as $\mathrm{SCP}_{\max }$ and $\mathrm{SCP}_{80 \%}$ were calculated by the kinetic measurement data (Fig. 5f) at different boundary conditions for ADC applications (Supplementary Table 5), and more details can be found in Supplementary Note 3.

\section{Computational details}

First-principles calculations were performed within the framework of density functional theory (DFT) and the plane-wave pseudopotential functional approach ${ }^{48,49}$, as implemented in the Vienna ab initio Simulation Package (VASP) ${ }^{50,51}$. Ion-electron interactions were implemented with the projectoraugmented wave (PAW) method ${ }^{52}$. The generalized gradient approximation (GGA) and the PBE functional ${ }^{53}$ were used with a $450 \mathrm{eV}$ limit for the plane-waves. All the atom positions were relaxed until the energy and force changes on each atom were less than $0.001 \mathrm{meV}$ and $0.01 \mathrm{eV} \cdot \AA^{-1}$, respectively. For geometry optimization and electronic structure calculations, the Brillouin zone was sampled with 2 ' 4 ' 4 $\Gamma$-centered Monkhorst-Pack K-point grid. Based on the self-consistent charge and potential, the Density Derived Electrostatic and Chemical (DDEC) net atomic charges are calculated (Supplementary Table $10)^{54}$. Grand canonical Monte Carlo (GCMC) simulations were employed to calculate the water adsorption in the AIPO at $303 \mathrm{~K}$. The framework structure was constructed from their corresponding experimental single-crystal diffraction data ${ }^{39}$. The material was treated as rigid frameworks with atoms 
frozen at their crystallographic positions, and the simulation box was made of 12 conventional unit cells $(2 \times 2 \times 3)$. A cutoff radius was set to $1.2 \mathrm{~nm}$ for the $L J$ interactions, while the long-range electrostatic interactions were handled by the Ewald summation technique. The augmented CVFF force field parameter ${ }^{55}$ was adopted to describe the LJ parameters for the atoms in the AIPOs framework, whereas water molecules were described by the simple SPC potential model ${ }^{56}$. This combination has been proven to be effective for the calculation of water sorption isotherm in zeolite 57,58 . Periodic boundary conditions were considered in all three dimensions. For each state point, GCMC simulations consisted of $2 \times 10^{8}$ steps to ensure the equilibration, followed by $3 \times 10^{8}$ steps to sample the desired thermodynamic properties. In addition, to obtain accurate ensemble averages in GCMC simulations, at least millions of configurations generated by random translation, rotation, regrowth, and swap moves are sampled in each simulation.

\section{Declarations}

\section{Competing Interests}

The authors declare no competing interests.

\section{Author contributions}

L.Z.L contributed to the synthesis, shaping and general characterization of EMM-8, collected watersorption data, calculated the thermodynamic efficiency of EMM-8 for water sorption, evaluated specific cooling power values, and contributed to the writing of the paper.

\section{Acknowledgments}

This work was supported by the National Natural Science Foundation of China (No. 51836009) and the National Key R\&D Program of China (No. 2018YFE0100300).

\section{References}

1. IEA Fuel Report: Renewable Energy Policies in a Time of Transition: Heating and Cooling (International Energy Agency, 2020).

2. Yalew, S. G. et al. Impacts of climate change on energy systems in global and regional scenarios. Nat Energy 5, 794-802 (2020).

3. D'Oca, S., Hong, T. Z. \& Langevin, J. The human dimensions of energy use in buildings: A review. Renew. Sust. Energ. Rev. 81, 731-742 (2018).

4. Lake, A., Rezaie, B. \& Beyerlein, S. Review of district heating and cooling systems for a sustainable future. Renew. Sust. Energ. Rev. 67, 417-425 (2017).

5. Ürge-Vorsatz, D., Cabeza, L. F., Serrano, S., Barreneche, C. \& Petrichenko, K. Heating and cooling energy trends and drivers in buildings. Renew. Sust. Energ. Rev. 41, 85-98 (2015). 
6. IEA Technology Report: The Future of Cooling (International Energy Agency, 2020).

7. Pinheiro, J. M., Salústio, S., Rocha, J., Valente, A. A. \& Silva, C. M. Adsorption heat pumps for heating applications. Renew. Sust. Energ. Rev. 119, 109528 (2020).

8. Cabeza, L. F. \& Schossig, P. Advances in sorption systems for energy effificient heating and cooling. Renew. Energ.. 110, 1-2 (2017).

9. Wang, R. Z. \& Oliveira, R. G. Adsorption refrigeration-An effificient way to make good use of waste heat and solar energy. Prog. Energy Combust. Sci. 32, 424-458 (2006).

10. Shmroukh, A. N., H. Ali, A. H. \& Ookawara, S. Adsorption working pairs for adsorption cooling chillers: A review based on adsorption capacity and environmental impact. Renew. Sust. Energ. Rev. 50, 445456 (2015)

11. Aristov, Y. I. Challenging offers of material science for adsorption heat transformation: A review. Appl. Therm. Eng. 50, 1610-1618 (2013).

12. Pan, Q. W., Peng, J. J. \& Wang R. Z. Application analysis of adsorption refrigeration system for solar and data center waste heat utilization. Energy Convers. Manage. 228, 113564 (2021).

13. Pan, Q. W., Peng, J. J., Wang, H. B., Sun, H. Q. \& Wang, R. Z. Experimental investigation of an adsorption air-conditioner using silica gel water working pair. Sol. Energy. 185, 64-71 (2019).

14. Wahono, S. K. et al. Plasma activation on natural mordenite-clinoptilolite zeolite for water vapor adsorption enhancement. Appl. Surf. Sci. 483, 940-946 (2019).

15. Ammann, J., Ruch, P., Michel, B. \& Studart, A. R. High-power adsorption heat pumps using magnetically-aligned zeolite structures. ACS Appl. Mater. Interfaces 11, 24037-24046 (2019).

16. Liu, X. L., Wang, X. R. \& Kapteijn, F. Water and metal-organic frameworks: from interaction toward utilization. Chem. Rev. 120, 8303-8377 (2020).

17. Canivet, J., Fateeva, A., Guo, Y., Coasne, B. \& Farrusseng, D. Water adsorption in MOFs: fundamentals and applications. Chem. Soc. Rev. 43, 5594-5617 (2014).

18. de Lange, M. F., Verouden, K. J. F. M., Vlugt, T. J. H., Gascon, J. \& Kapteijn, F. Adsorptiondriven heat pumps: the potential of metal-organic frameworks. Chem. Rev. 115, 12205-12250 (2015).

19. Furukawa, H. et al. Water adsorption in porous metal-organic frameworks and related materials. J. Am. Chem. Soc. 136, 4369-4381 (2014).

20. Fathieh, F. et al. Practical water production from desert air. Sci. Adv. 4, 3198 (2018).

21. Wang, S. et al. A robust large-pore zirconium carboxylate metal-organic framework for energyefficient water-sorption-driven refrigeration. Nat Energy 3, 985-993 (2018).

22. Cadiau, A. et al. Design of hydrophilic metal organic framework water adsorbents for heat reallocation. Adv. Mater. 27, 4775-4780 (2015).

23. Lenzen D. et al. A metal-organic framework for effificient water-based ultra-low-temperature-driven cooling. Nat Commun 10, 3025 (2019). 
24. Cho, K. H. et al. Defective Zr-fumarate MOFs enable high-efficiency adsorption heat allocations. ACS Appl. Mater. Interfaces 13, 1723-1734 (2021).

25. Cho, K. H. et al. Rational design of a robust aluminum metal-organic framework for multi-purpose water-sorption-driven heat allocations. Nat Commun 11, 5112 (2020).

26. Kummer, $\mathrm{H}$. et al. A functional full-scale heat exchanger coated with aluminum fumarate metal-organic framework for adsorption heat transformation. Ind. Eng. Chem. Res. 56, 8393-8398 (2017).

27. Lenzen, D. et al. Scalable green synthesis and full-scale test of the metal-organic framework CAU10-H for use in adsorption-driven chillers. Adv. Mater. 30, 1705869 (2018).

28. Ryu, U. J. et al. Recent advances in process engineering and upcoming applications of metal-organic frameworks. Coord. Chem. Rev. 426, 213544 (2021).

29. DeSantis, D. et al. Techno-economic analysis of metal-organic frameworks for hydro-gen and natural gas storage. Energy Fuels 31, 2024-2032 (2017).

30. Kim, S. H. et al. Improvement of cooling performance of water adsorption chiller by using aluminophophate adsorbent. Microporous Mesoporous Mater. 309, 110572 (2020).

31. Freni, A. et al. SAPO-34 coated adsorbent heat exchanger for adsorption chillers. Appl. Therm. Eng. 82, 1-7 (2015).

32. Li, A., Ismail, A. B., Thu, K., Ng, K. C. \& Loh, W. S. Performance evaluation of a zeolite-water adsorption chiller with entropy analysis of thermodynamic insight. Appl. Energy 130, 702-711 (2014).

33. Youssef, P. G., Mahmoud, S. M. \& AL-Dadah, R. K. Performance analysis of four bed adsorption water desalination/ refrigeration system, comparison of AQSOA-ZO2 to silica-gel. Desalination $\mathbf{3 7 5 , 1 0 0 -}$ 107 (2015).

34. Rocky, K. A., Pal, A., Rupam, T. H., Nasruddin. \& Saha, B. B. Zeolite-graphene composite adsorbents for next generation adsorption heat pumps. Microporous Mesoporous Mater. 313, 110839 (2021).

35. Mitsubishi Plastics. Zeolitic Water Vapor Adsorbent: AQSOA. http://www.aaasaveenergy.com/products/001/pdf/AQSOA_1210E.pdf (accessed 5 July 2021).

36. McCague, C., Huttema, W., Fradin, A. \& Bahrami, M. Lab-scale sorption chiller comparison of FAMZ02 coating and pellets. Appl. Therm. Eng. 173, 115219 (2020).

37. Krajnc, A. et al. Superior performance of microporous aluminophosphate with LTA topology in solarEnergy storage and heat reallocation. Adv. Energy Mater. 7, 1601815 (2017).

38. Yang, M., Fan, D., Wei, Y. X., Tian, P. \& Liu, Z. M. Recent progress in methanol-to-olefins (MTO) catalysts. Adv. Mater. 31, 1902181 (2019).

39. Cao, G., Afeworki, M., Kennedy, G. J., Strohmaier, K. G. \& Dorset, D. L. Structure of an aluminophosphate EMM-8: a multi-technique approach. Acta Cryst. B63, 56-62 (2007).

40. Afeworki, M., Cao, G., Dorset, D. L., Strohmaier, K. G. \& Kennedy, G. J. Multinuclear and multidimensional solid-state NMR characterization of EMM-8. Microporous Mesoporous Mater. 103, 216-224 (2007). 
41. Pérez-Carvajal, J., Boix, G., Imaz, I. \& Maspoch, D. The imine-based COF TpPa-1 as an efficient cooling adsorbent that can be regenerated by heat or light. Adv. Energy Mater. 9, 1901535 (2019).

42. Lee, J. S. et al. The porous metal-organic framework CUK-1 for adsorption heat allocation toward green applications of natural refrigerant water. ACS Appl. Mater. Interfaces. 11, 25778-25789 (2019).

43. Buchholz, A., Wang, W., Arnold, A., Xu, M. \& Hunger, M. Successive steps of hydration and dehydration of silicoaluminophosphates H-SAPO-34 and H-SAPO-37 investigated by in situ CF MAS NMR spectroscopy. Microporous Mesoporous Mater. 57, 157-168 (2003).

44. Kolokathis, P. D., Pantatosaki, E. \& Papadopoulos, G. K. Modeling the hydrationinduced structural transitions of the SAPO-34 zeolite and their impact on the water's sorbed phase equilibrium and dynamics. J. Phys. Chem. C 124, 11480-11489 (2020).

45. Varlec, J. et al. Dehydration of $\mathrm{AlPO}_{4}-34$ studied by variable-temperature NMR, XRD and firstprinciples calculations. New J. Chem. 40, 4178-4186 (2016).

46. Aristov, Y. Concept of adsorbent optimal for adsorptive cooling/ heating. Appl. Therm. Eng. 72, 166175 (2014).

47. Sapienza, A., Santamaria, S., Frazzica, A., Freni, A. \& Aristov, Y. I. Dynamic study of adsorbers by a new gravimetric version of the Large Temperature Jump method. Appl. Energy. 113, 1244-1251 (2014).

48. Rajagopal, A. K. \& Callaway, J. Inhomogeneous electron gas. Phys. Rev. B 7, 1912-1919 (1973).

49. Kohn, W. \& Sham, L. J. Self-consistent equations including exchange and correlation effects. Phys. Rev. 140, A1133 (1965).

50. Kresse, G. \& Furthmüller, J. Efficient iterative schemes for ab initio total-energy calculations using a plane-wave basis set. Phys. Rev. B 54, 11169-11186 (1996).

51. Kresse, G. Ab initio molecular dynamics for liquid metals. Phys. Rev. B 48, 13115-13118 (1993).

52. Kresse, G. \& Joubert, D. From ultrasoft pseudopotentials to the projector augmented-wave method. Phys. Rev. B 59, 1758-1775 (1999).

53. Perdew, J. P., Burke, K. \& Ernzerhof, M. Generalized gradient approximation made simple. Phys. Rev. Lett. 78, 1396 (1997).

54. Manz, T. A. \& Limas, N. G. Introducing DDEC6 atomic population analysis: part 1. Charge partitioning theory and methodology. RSC Adv. 6, 47771-47801 (2016).

55. Channon, Y. M., Catlow, C. R. A., Gorman, A. M. \& Jackson, R. A. Grand canonical monte carlo investigation, of water adsorption in heulandite-type zeolites. J. Phys. Chem. B 102, 4045-4048 (1998).

56. Mark, P. \& Nilsson L. Structure and dynamics of the TIP3P, SPC, and SPC/E water models at 298 K. J. Phys. Chem. A 105, 9954-9960 (2001).

57. Hill, J. R., Minihan, A. R., Wimmer, E. \& Adams, C. J. Framework dynamics including computer simulations of the water adsorption isotherm of zeolite Na-MAP. Phys. Chem. Chem. Phys. 2, 4255- 
$4264(2000)$.

58. Henninger, S. K., Schmidt, F. P., Nunez, T. \& Henning, H. M. Monte carlo investigations of the water adsorption behavior in MFI type zeolites for different $\mathrm{Si} / \mathrm{Al}$ ratios with regard to heat storage applications. Adsorption 11, 361-366 (2005).

\section{Figures}

a

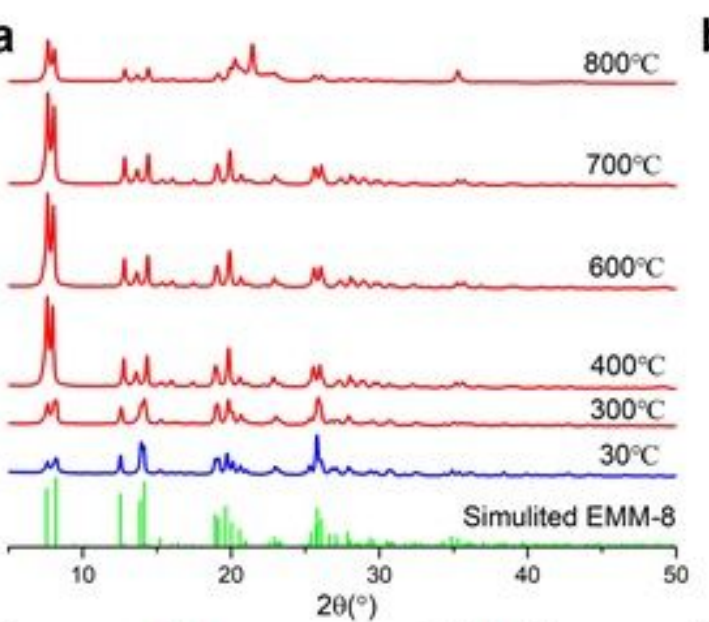

C

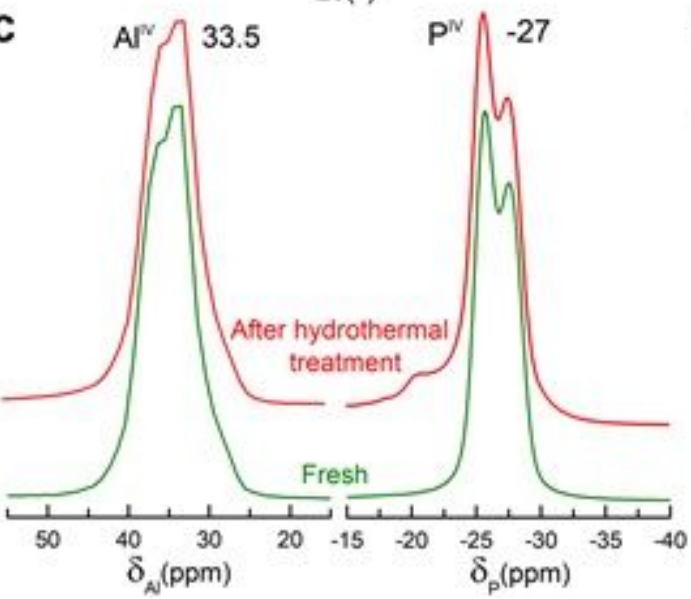

b
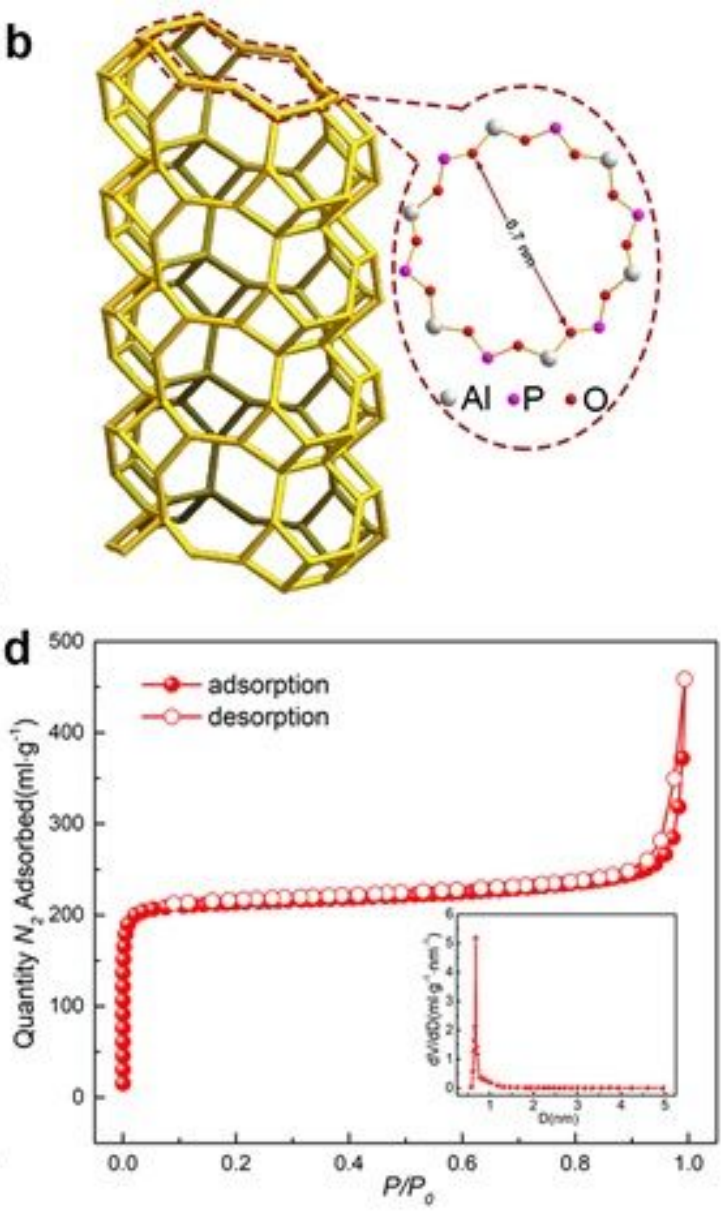

Figure 1

Structure, texture, and hydrothermal stability of EMM-8. a Temperature-dependent in-situ powder X-ray diffraction of EMM-8. b Structure schematic of the 12-ring channel of EMM-8. c 27Al and 31P MAS NMR spectra of EMM-8 for the fresh calcined sample (green) and the same sample after hydrothermal treatment (red). $d$ Nitrogen sorption isotherms of the calcined EMM-8 taken at $77 \mathrm{~K}$, the inset image gives the pore size distribution of the sample. 
a

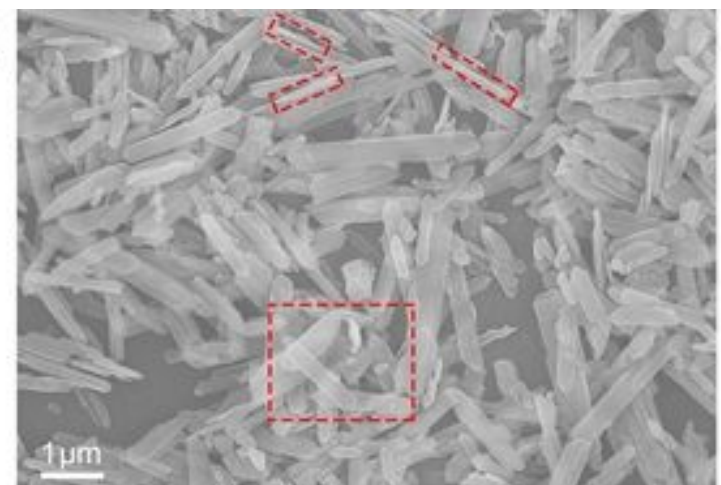

C

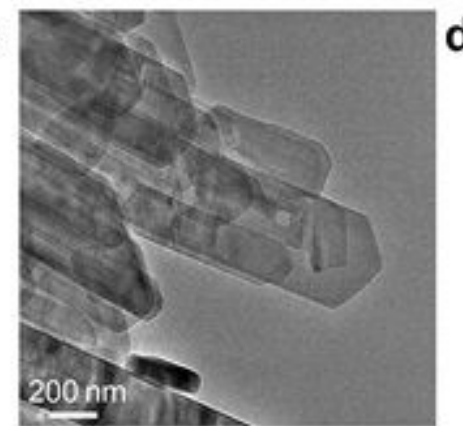

d

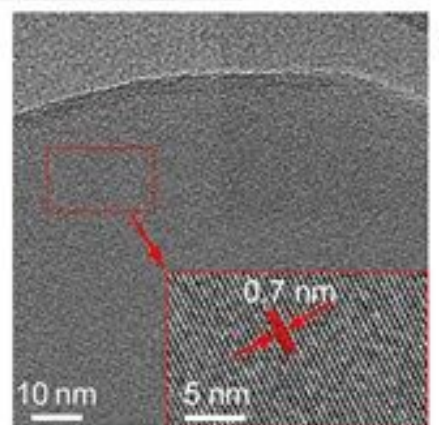

f

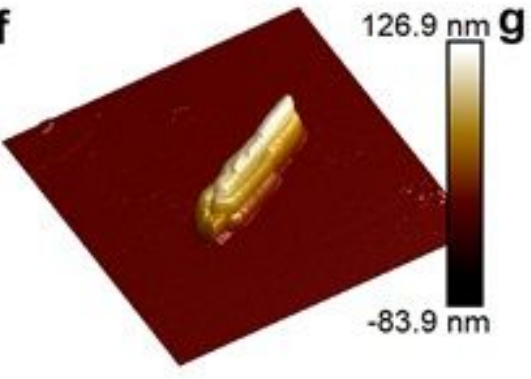

b
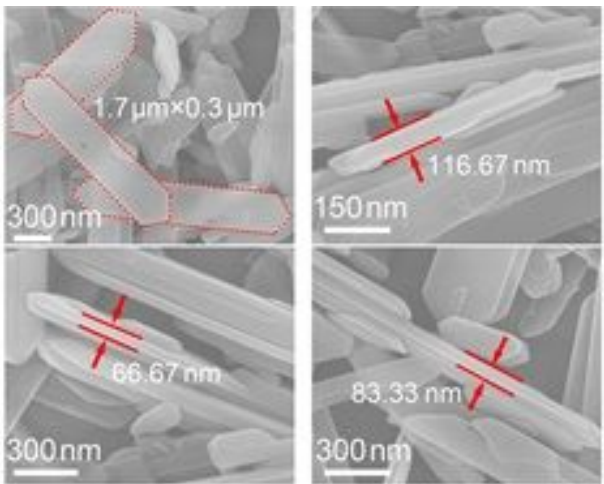

e
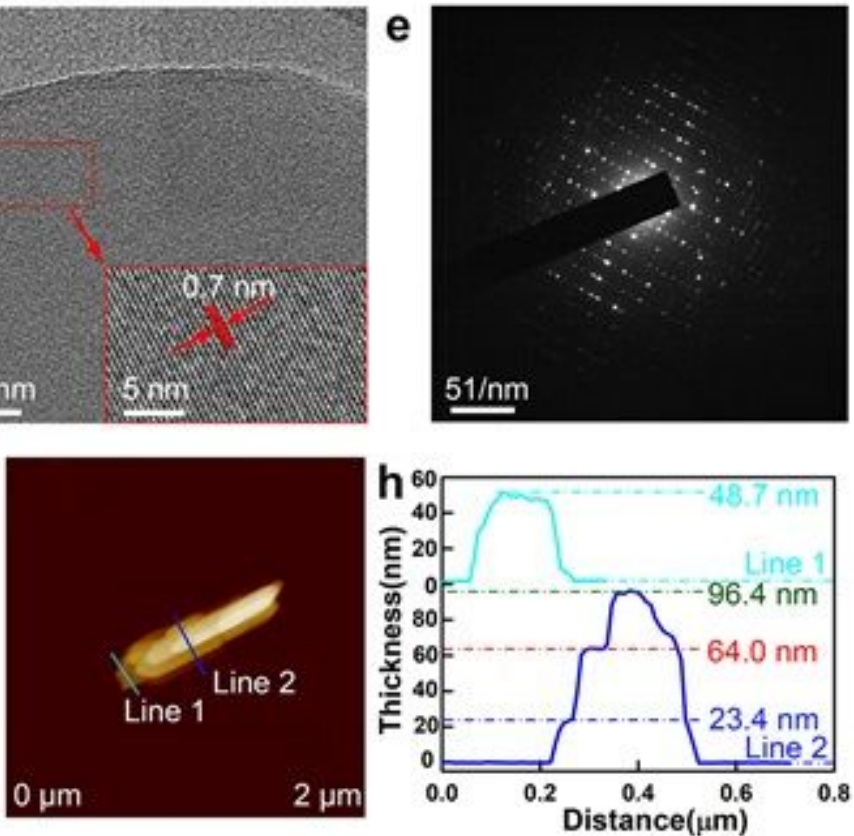

Figure 2

Morphologic characterization of the as-obtained EMM-8. a, b SEM images of the EMM-8 with different magnification. c-e TEM images of the EMM-8 with different magnification and its SAED patterns. $f-h$ AFM images of the EMM-8. 

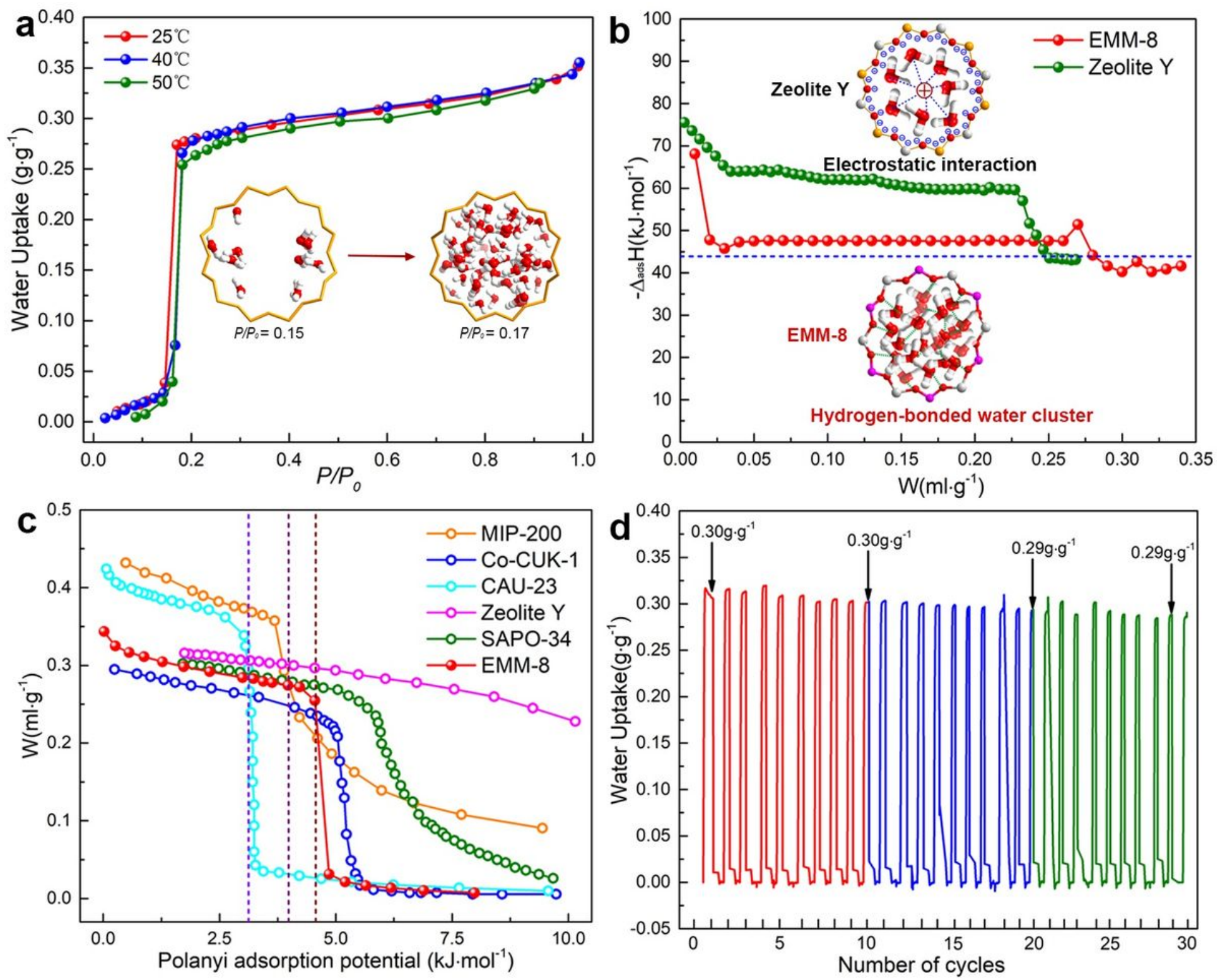

Figure 3

Water adsorption properties of EMM-8. a Water adsorption isotherms recorded at three different temperatures. $b$ The calculated adsorption enthalpy as a function of water loading. The blue dash line represents the water evaporation enthalpy. c Characteristic curves determined by adsorption isotherm at $30^{\circ} \mathrm{C}$ of EMM-8 and other reference materials. The dashed lines represent the optimal adsorption potential for cooling to $10^{\circ} \mathrm{C}$ from $30^{\circ} \mathrm{C}$ (black), cooling to $5^{\circ} \mathrm{C}$ from $30^{\circ} \mathrm{C}$ (purple), and heating to $45^{\circ} \mathrm{C}$ from $15{ }^{\circ} \mathrm{C}$ (red). d Water adsorption/desorption cycling performance of EMM-8. Test conditions: adsorption at $30{ }^{\circ} \mathrm{C}$ with $30 \% \mathrm{RH}$, and desorption at $110{ }^{\circ} \mathrm{C}$ under vacuum. 

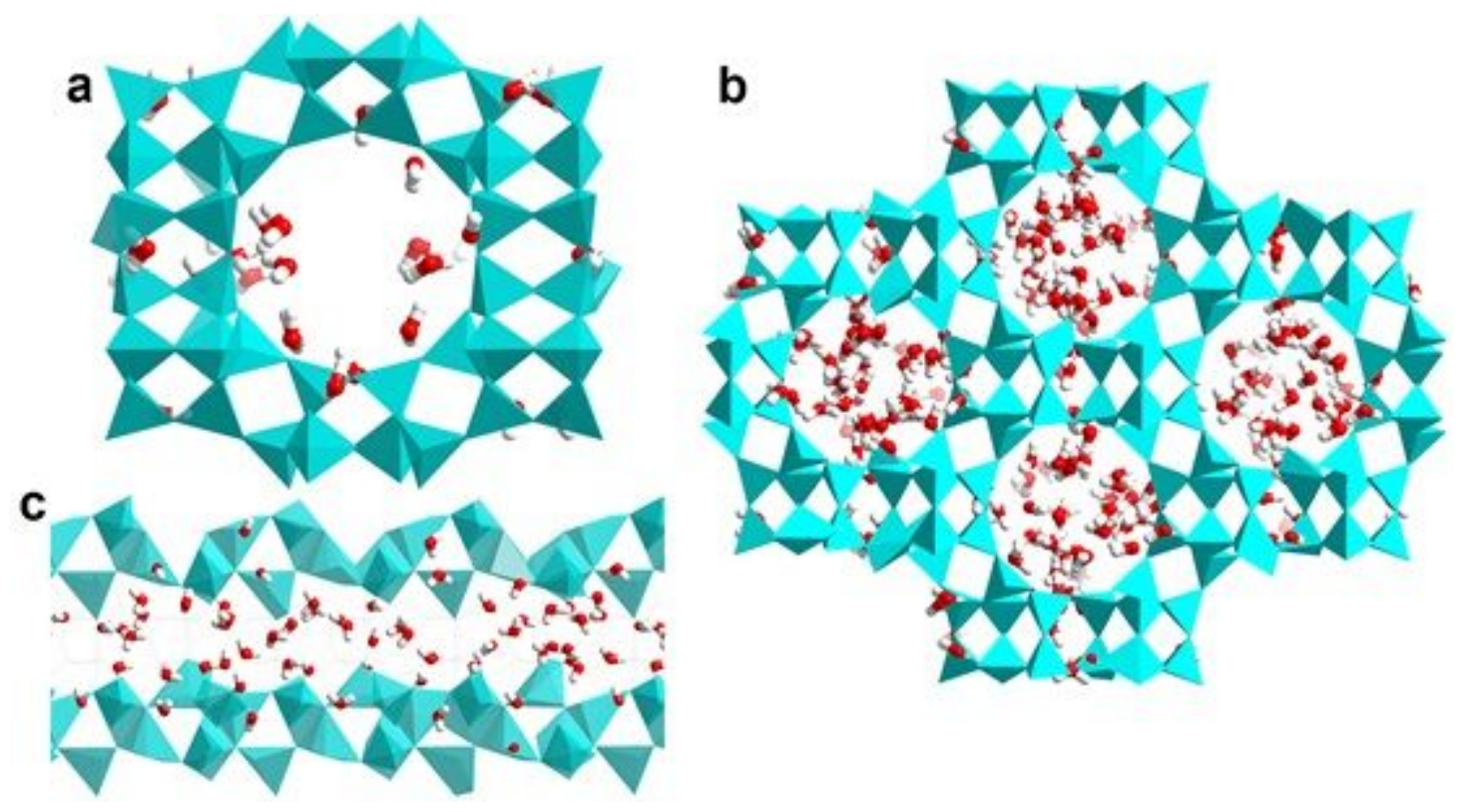

Figure 4

GCMC simulation results of water adsorption. a The adsorbed water molecules coordinating to aluminum atoms in $\mathrm{EMM}-8$ at low pressure $(\mathrm{P} / \mathrm{P} 0=0.01)$. $b$ Aggregation of hydrogen-bonded water molecules in the 12-ring channel at $\mathrm{P} / \mathrm{PO}=0.3$ from the top view. $\mathrm{c}$ The cross-section of the water-loaded channel.
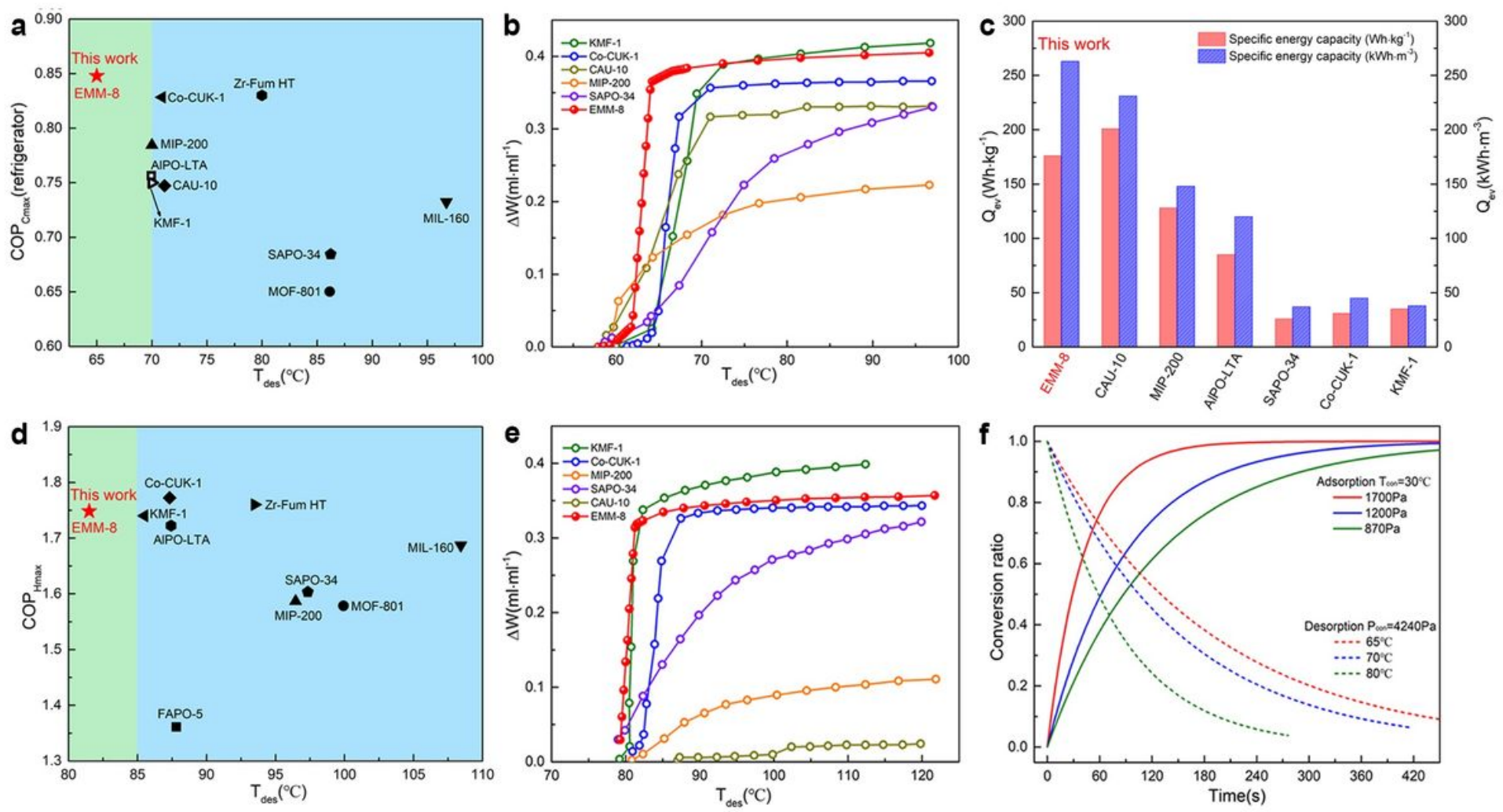

Figure 5

Performance evaluation of EMM-8 in comparison with other adsorbents. a Comparison of maximum COP values and their corresponding driven temperature for cooling of EMM-8 and reference materials. 
Refrigeration conditions used were $\operatorname{Tev}=5^{\circ} \mathrm{C}$ and $\operatorname{Tcon}=30^{\circ} \mathrm{C}$. b Plots of the volumetric working capacities under the varying regeneration temperatures for $A D C$ conditions $\left(\mathrm{Tev}=5^{\circ} \mathrm{C}\right.$ and $\left.\mathrm{Tcon}=30^{\circ} \mathrm{C}\right)$. c Specific energy capacities for EMM-8 and benchmark adsorbents expressed in gravimetric and volumetric scales $\left(\mathrm{Tev}=10^{\circ} \mathrm{C}\right.$, Tcon $=30^{\circ} \mathrm{C}$, and Tdes $\left.=65^{\circ} \mathrm{C}\right)$. $\mathrm{d}$ Maximum COP values versus the driven temperature for heat pump application. The conditions used for calculation were Tev $=15^{\circ} \mathrm{C}$ and Tcon $=45^{\circ} \mathrm{C}$. e Plots of the volumetric working capacities under the varying regeneration temperatures for AHP conditions $\left(\mathrm{Tev}=15^{\circ} \mathrm{C}\right.$ and $\left.\mathrm{Tcon}=45^{\circ} \mathrm{C}\right)$. $\mathrm{f}$ Dimensionless water uptake curves during adsorption and desorption run under varying conditions. The size of EMM-8 grain is $0.45 \sim 0.6 \mathrm{~mm}$.

\section{Supplementary Files}

This is a list of supplementary files associated with this preprint. Click to download.

- SI20210705.doc 